\title{
Stent-Assisted Coiling of Ruptured Wide-Necked Intracranial Aneurysms
}

\author{
CONGHUI LI ${ }^{1,2}$, YOUXIANG LI ${ }^{1}$ \\ ${ }^{1}$ Beijing Neurosurgical Institute and Beijing Tiantan Hospital, Capital Medical University; Beijing, China \\ ${ }^{2}$ The first Hospital of Shijiazhuang, Shijiazhuang; Hebei, P.R. China
}

Key words: cerebral aneurysm, coil, stent, subarachnoid hemorrhage

\section{Summary}

This study aimed to report the results and outcome of stent-assisted coiling of ruptured widenecked intracranial aneurysms. We retrospectively reviewed 19 consecutive patients (11 men, eight women; mean age, 59.5 years; range, 43-78 years) with acutely ruptured wide-necked intracranial aneurysms who were treated with stentassisted coil embolization. The mean length of angiographic follow-up was 5.2 months (range, 3-10 months). There was no technique-related complication and the 30-day mortality rate was $10.5 \%$ (two of 19). There was one case of rebleeding, and clinical outcome was poor for the patient $(5.3 \%$ [one of 19] who had a Glasgow Outcome Scale score of 2 at the end of the study period). Stent-assisted coiling is a feasible treatment for ruptured wide-necked intracranial aneurysms that are difficult to treat surgically or with balloon-assisted embolization.

\section{Introduction}

The published data on stent-assisted coiling results in patients with ruptured aneurysms are limited and the mortality and morbidity of the procedure are still $14 \%-22 \%{ }^{1-4}$. This retrospective study reports another series of stent-assisted coiling for ruptured wide-necked intracranial aneurysms.

\section{Materials and Methods}

We retrospectively reviewed 19 consecutive patients $(20 \%$ of the total number of ruptured aneurysms treated during the study period) with ruptured wide-necked intracranial aneurysms who were treated with stent-assisted coiling between January 2010 and January 2012. There were 11 men and eight women with a mean age of 59.5 years (range, 43-78 years). Endovascular treatment was usually favored over surgical clipping in older patients, those in poor condition and patient preference. The primary occlusion rate was classified as "total occlusion" ( $>95 \%$ occlusion rate), "neck remnant" (>80\% occlusion rate with contrast medium filling only the neck area of the aneurysm), "partial occlusion" (distal filling of contrast medium in the aneurysm regardless of the occlusion rate), or "none" (unsuccessful coiling). Angiographic occlusion was considered to be adequate if either complete occlusion of the aneurysm or a small neck remnant with over $80 \%$ occlusion was achieved.

Ruptured wide-necked intracranial aneurysms may be treated by stent-assisted coiling if additional support or remodeling of the aneurysm neck is required. Major complications were considered to constitute technical failures, regardless of angiographic outcome. The preoperative clinical condition of the patients was evaluated using the Hunt and Hess classification (5) and the clinical outcome using the Glasgow Outcome Scale (6).

The angiographic characteristics of the ruptured aneurysms are listed in Table 1 . Six aneurysms were located in the posterior circulation, and one patient had two aneurysms. One of the ruptured vertebral artery aneurysms was considered to be a dissecting aneurysm. All of the aneurysms had a neck width of more than 4 $\mathrm{mm}$ or a dome-to-neck ratio of 1.5 or less and additional support or remodeling of the aneurysm neck was required. 

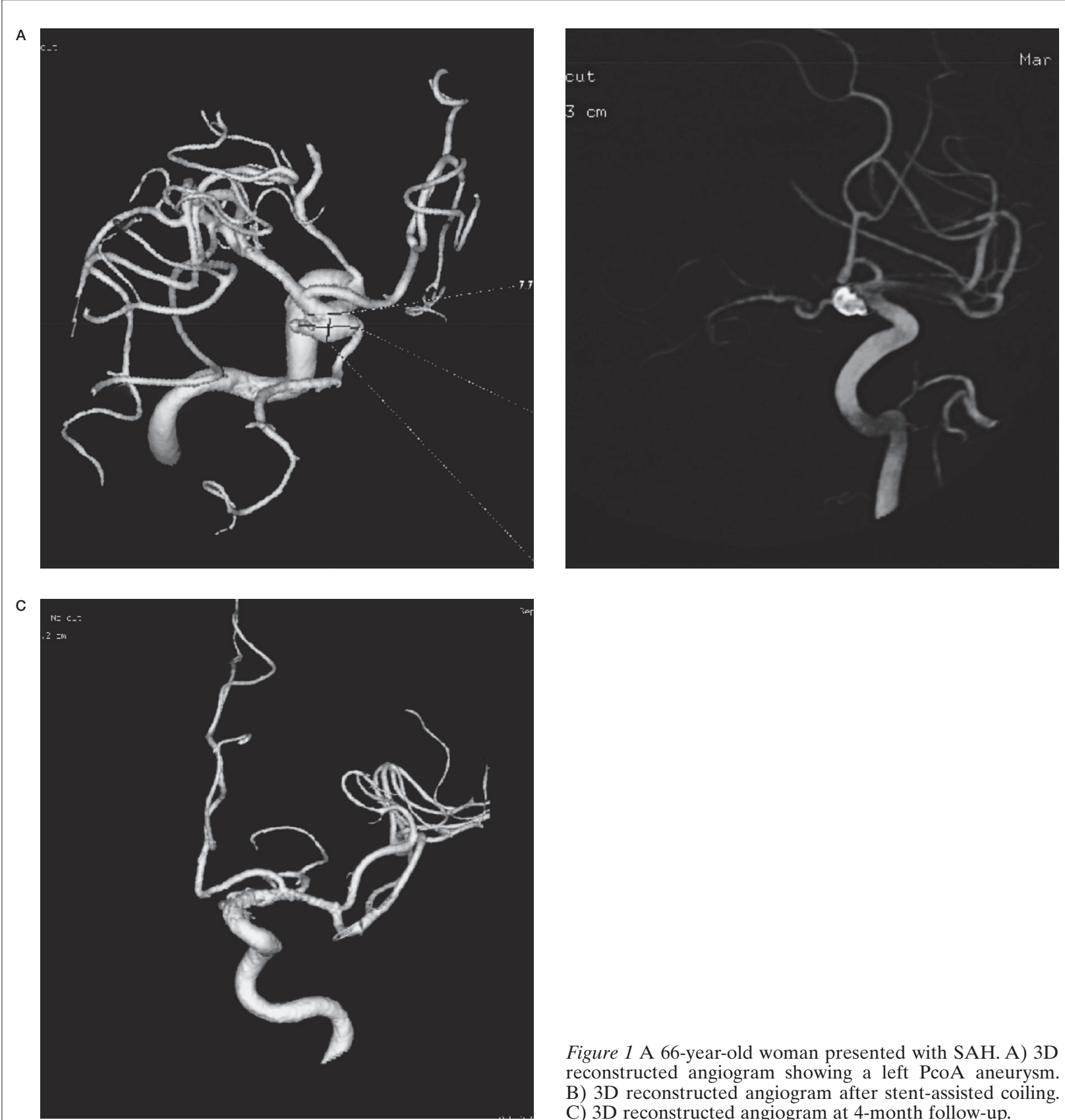

Figure 1 A 66-year-old woman presented with SAH. A) 3D reconstructed angiogram showing a left PcoA aneurysm. B) 3D reconstructed angiogram after stent-assisted coiling. C) 3D reconstructed angiogram at 4-month follow-up.

\section{Procedure and Medication}

Selective angiograms and three-dimensional angiograms were evaluated in detail before the embolization procedure. Endovascular treatment of the aneurysm was performed between one and 25 days (median three days) after aneurysmal rupture. A $90-\mathrm{cm}$ guiding catheter (Boston Scientific, Fremont, CA, USA) was inserted into the relevant carotid or vertebral artery. A coil delivery microcatheter (Echelon-10,
Covedian) was first navigated into the aneurysm sac followed by navigating a self-expandable stent (Enterprise; Cordman) into the parent vessel and delivering the stent. Once the stent had been delivered, subsequent coil embolization was performed to obtain circulatory exclusion of the lesion. The microcatheter was trapped by the stent during coiling. Aneurysms were packed with either Microplex detachable coils (Microvention, Aliso Vijo) or Micrus coils (Ev3, M.T.I) or with a combination of the two 
Table 1 Ruptured aneurysms treated with stent-assisted coil embolization.

\begin{tabular}{|c|c|c|c|c|c|c|c|c|c|c|c|}
\hline $\begin{array}{l}\text { Patient } \\
\text { No. }\end{array}$ & Sex & Age & $\begin{array}{l}\text { H-H } \\
\text { Grade }\end{array}$ & $\begin{array}{l}\text { Days } \\
\text { after } \\
\text { SAH }\end{array}$ & Locations & $\begin{array}{l}\text { Aneurysm } \\
\text { size }(\mathrm{mm})\end{array}$ & $\begin{array}{l}\text { Neck size } \\
(\mathrm{mm})\end{array}$ & Results & Complications & $\begin{array}{l}\text { Follow-up } \\
\text { months) }\end{array}$ & $\begin{array}{l}\text { Outcome } \\
\text { (GOS) }\end{array}$ \\
\hline 1 & M & 58 & 3 & 25 & $\mathrm{BA}$ & $2.5 \times 4$ & 4 & $\begin{array}{l}\text { Total } \\
\text { occlusion }\end{array}$ & No & 10 & 5 \\
\hline 2 & M & 46 & 1 & 7 & $\begin{array}{l}\text { Left } \\
\text { ophthalmic } \\
\text { ICA }\end{array}$ & $4.8 \times 4.9$ & 4.3 & $\begin{array}{l}\text { Partial } \\
\text { occlusion }\end{array}$ & No & 6 & 5 \\
\hline 3 & $\mathrm{~F}$ & 78 & 3 & 2 & Right VA & $7.8 \times 6.9$ & 5.3 & $\begin{array}{l}\text { Total } \\
\text { occlusion }\end{array}$ & No & 6 & 4 \\
\hline 4 & M & 43 & 3 & 2 & Right VA & $4.5 \times 2.7$ & 4 & $\begin{array}{l}\text { Total } \\
\text { occlusion }\end{array}$ & No & 3 & 5 \\
\hline 5 & $\mathrm{~F}$ & 78 & 1 & 2 & $\mathrm{BA}$ & $4.2 \times 5.0$ & 4.5 & $\begin{array}{l}\text { Total } \\
\text { occlusion }\end{array}$ & No & 7 & 5 \\
\hline 7 & M & 55 & 1 & 10 & BA & $11 \times 12$ & 7 & $\begin{array}{l}\text { Total } \\
\text { occlusion }\end{array}$ & $\begin{array}{l}\text { Paralysis } \\
\text { of abducens } \\
\text { nerve }\end{array}$ & 3 & 4 \\
\hline 8 & M & 55 & 2 & 3 & $\begin{array}{l}\text { Left } \\
\text { PCoA }\end{array}$ & $1.5 \times 1.7$ & 1.3 & $\begin{array}{l}\text { Partial } \\
\text { occlusion }\end{array}$ & No & 3 & 5 \\
\hline 9 & $\mathrm{~F}$ & 60 & 4 & 1 & $\begin{array}{l}\text { Left } \\
\text { PCoA }\end{array}$ & $8.3 \times 7.5$ & 6.5 & $\begin{array}{l}\text { Total } \\
\text { occlusion }\end{array}$ & No & Death & - \\
\hline 10 & M & 56 & 1 & 15 & $\begin{array}{l}\text { Right } \\
\text { PCoA }\end{array}$ & $2.0 \times 2.5$ & 2.3 & $\begin{array}{l}\text { Total } \\
\text { occlusion }\end{array}$ & No & 3 & 5 \\
\hline 11 & $\mathrm{~F}$ & 57 & 3 & 3 & $\begin{array}{l}\text { Right } \\
\text { PCoA }\end{array}$ & $1.6 \times 2.0$ & 2.0 & $\begin{array}{l}\text { Partial } \\
\text { occlusion }\end{array}$ & $\begin{array}{l}\text { Rebleeding } \\
\text { on } 5 \text { days }\end{array}$ & 4 & 1 \\
\hline 14 & $\mathrm{~F}$ & 72 & 2 & 4 & $\begin{array}{l}\text { Bilateral } \\
\text { PCoA }\end{array}$ & $\begin{array}{l}4.7 \times 5.5 \\
2.0 \times 2.5\end{array}$ & $4.0 ; 1.5$ & $\begin{array}{l}\text { Total } \\
\text { occlusion; } \\
\text { neck } \\
\text { remnant }\end{array}$ & No & 5 & 5 \\
\hline 15 & $\mathrm{~F}$ & 63 & 2 & 3 & $\begin{array}{l}\text { Left } \\
\text { PCoA }\end{array}$ & $0.75 \times 1.5$ & 1.2 & $\begin{array}{l}\text { Partial } \\
\text { occlusion }\end{array}$ & No & 3 & 5 \\
\hline 16 & $\mathrm{M}$ & 66 & 3 & 2 & Left VA & $4.3 \times 5.2$ & 4.0 & $\begin{array}{l}\text { Neck } \\
\text { remnant }\end{array}$ & No & 6 & 5 \\
\hline 17 & M & 58 & 2 & 10 & $\begin{array}{l}\text { Left } \\
\text { PCoA }\end{array}$ & $3.5 \times 4.0$ & 2.8 & $\begin{array}{l}\text { Neck } \\
\text { remnant }\end{array}$ & No & 10 & 5 \\
\hline 18 & M & 54 & 2 & 3 & $\begin{array}{l}\text { Right } \\
\text { PCoA }\end{array}$ & $1.8 \times 1.6$ & 1.5 & $\begin{array}{l}\text { Total } \\
\text { occlusion }\end{array}$ & $\begin{array}{l}\text { In-stent } \\
\text { thrombosis }\end{array}$ & 3 & 5 \\
\hline 19 & $\mathrm{~F}$ & 47 & 2 & 4 & $\begin{array}{l}\text { Right } \\
\text { ophthalmic } \\
\text { ICA }\end{array}$ & $4.5 \times 3.4$ & 3.5 & $\begin{array}{l}\text { Partial } \\
\text { occlusion }\end{array}$ & No & 7 & 5 \\
\hline
\end{tabular}

(Figure 1); 3000 IU of heparin was administered intravenously after the first coil deployment. A loading dose of $300 \mathrm{mg}$ Clopidogrel and $300 \mathrm{mg}$ Aspirin was given orally or through a nasogastric tube two hours before treatment and $100 \mathrm{mg}$ Aspirin daily for three to six months was started immediately after the procedure.

\section{Follow-up}

The mean duration of angiographic followup was 5.2 months (range, 3-10 months). The first follow-up angiography was performed depending on the primary result of the endovascular treatment. 


\section{Results}

The median Hunt Hess score was 2 (range, 1-4), and the median Fisher grade was 3 (range, 1-4). Thirteen patients were treated for aneurysms in the anterior circulation.

The majority of these aneurysms were internal carotid artery (ICA)-posterior communicating aneurysms $(N=11)$ and two patients were treated for aneurysms located in the ophthalmic ICA. Three patients were treated for ruptured vertebral artery aneurysms and three patients were treated for an aneurysm of the basilar artery.

Nearly all of the aneurysms treated had a dome-to-neck ratio less than 1.5 . The remaining six patients had a neck size greater than 4 $\mathrm{mm}$. No patient underwent placement of ventriculostomy prior to embolization and antiplatelet therapy. All endovascular techniques were successful, and an adequate primary angiographic result was achieved in 14 (73.7\%) of the 19 patients. There was no procedure-related focal vasospasm.

\section{Early Rebleeding}

There was one case $(5.3 \%)$ of early rebleeding in our series, which ensued after partial occlusion of a right ICA aneurysm. In this patient, an Enterprise stent was successfully deployed across the neck of the aneurysm, but there were considerable difficulties in subsequent coiling because of small size. Initially, the condition of the patient stabilized, but it deteriorated five days after the procedure and rebleeding of the aneurysm was observed at CT.

\section{In-Stent Thrombosis}

One $(5.3 \%)$ of the 19 patients experienced a thromboembolic complication during routine endovascular treatment of a ruptured aneurysm and was successfully treated by $5 \mathrm{mg}$ Tirophiban via the guiding catheter.

\section{Clinical Outcome}

The 30-day mortality rate was $10.5 \%$ (2 of 19). Sixteen $(84.2 \%)$ of the 19 patients had a Glasgow Outcome Scale score of 4 or 5 at follow-up.

\section{Discussion}

Ruptured wide-necked aneurysms can be difficult to treat with surgical clipping or balloon-assisted coiling, and stent-assisted coiling may offer an important alternative treatment. To our knowledge, few studies have described stent-assisted coiling for acute ruptured aneurysms ${ }^{1-4}$. We add another series to evaluate the role of stent-assisted coiling in acute ruptured aneurysms. The main problem of introducing a stent is the high risk of parent vessel occlusion which necessitates anticoagulation and antiplatelet therapy during and after the procedure. The frequency of this complication varies in the literature, but reported rates range from 2.5 to $28 \%{ }^{7}$. An intracranial stent is considered to be thrombogenic until the stent has been covered by endothelialization and the normal intrinsic fibrinolytic activity on the endothelium has renewed ${ }^{8}$. Therefore, deployment of a stent generally necessitates pre- and post-procedural treatment with antiplatelet agents. However, these drugs are contraindicated in cases of acute subarachnoid hemorrhage (SAH) and nonsecured aneurysms. Although there is not enough evidence to suggest that antiplatelet agents may be safely administered in patients with acutely ruptured aneurysms, administration of Clopidogrel and Aspirin several hours before stent-assisted coiling has been shown to decrease the rate of thromboembolic events ${ }^{9}$. Tirophiban was administered only in the event of thromboembolic complications. Preoperative or perioperative treatment with Tirophiban may increase the risk of rebleeding with acutely ruptured aneurysms, and our results suggest there is no need for routine administration of Tirophiban in stent-assisted endovascular treatment of patients with acute $\mathrm{SAH}$.

We did not encounter substantial neointimal hyperplasia or in-stent stenosis in our series. However, we do not know if this is attributable to the flexibility of the Enterprise stent, to minor endothelial injury, or to the properties of the intracranial vasculature. Another reason for the lack of in-stent stenosis may be the short follow-up period rather than the characteristics of the stent. The existing data regarding the use of intracranial stents are promising, but longer follow-up studies are needed to evaluate the dynamics and delayed effects of these stents on the intracranial vasculature. We encountered a somewhat higher complication 
rate than was reported in previous series of stent-assisted coil embolization of wide-necked intracranial aneurysms. Mocco et al. ${ }^{10}$ reported a series of 141 patients treated with stent-assisted (Enterprise; Cordis Neurovascular/Johnson and Johnson, Miami, FL, USA) embolization. A mortality rate of $2 \%$ (three of 141 ) was reported in that study, with a mortality rate of $12 \%$ (two of 16) in the ruptured aneurysm group. A primary occlusion rate of $90 \%$ or greater was reported in 81 (76\%) of the 107 aneurysms in which coiling procedures were attempted in that study. Our series, however, consisted of complicated ruptured aneurysms that were difficult to treat and were not considered suitable for surgical clipping or balloon-assisted embolization. Our morbidity and mortality rates are largely attributable to the severe nature of SAH. Our results are comparable to the baseline results set by the International Subarachnoid Aneurysm Trial, in which 250 (23.5\%) of 1063 patients with ruptured intracranial aneurysms allocated to undergo endovascular treatment were dead or dependent at one year ${ }^{11}$. There was one case of early re- bleeding in our series, and the clinical outcome at follow-up was good for most of our patients.

Early treatment of ruptured aneurysms is recommended because of the high risk of subsequent rerupture ${ }^{12,13}$. According to our midterm follow-up results, stent-assisted coil embolization is a feasible endovascular treatment option for ruptured wide-necked intracranial aneurysms that are difficult to treat with balloon-assisted embolization or surgical clipping. The optimal antiplatelet medication during acute-phase treatment has yet to be determined, and a longer follow-up series is needed to evaluate the long-term efficacy and safety of stent-assisted coil embolization during acute SAH.

\section{Conclusion}

Stent-assisted coil embolization appears to be an option when balloon remodelling or clipping are not possible but it does carry higher risks than coiling alone based on the results of this and previous studies. 


\section{References}

$1 \mathrm{Xu}$ F, Song D. Stent-assisted coiling in acutely ruptured intracranial aneurysms. Am J Neuroradiol. 2012; 33: E28.

2 Tone O, Tomita H, Tamaki M, et al. Stent-assisted coiling of an acutely ruptured large aneurysm of the internal carotid artery: case report. No Shinkei Geka. 20y; 34 (5): 5055-5011 [Article in Japanese].

3 Golshani K, Ferrel A, Lessne M, et al. Stent-assisted coil emboilization of ruptured intracranial aneurysms: A retrospective multicenter review. Surg Neurol Int. 2012; 3: 84

4 Cruz JP, O'Kelly C, Kelly M, et al. Pipeline embolization device in aneurysmal subarachnoid hemorrhage. Am J Neuroradiol. 2013; 34 (2): 271-276. Epub 2012 Oct 11 .

5 Hunt WE, Hess RM. Surgical risk as related to time of intervention in the repair of intracranial aneurysms. J Neurosurg. 1968; 28: 14-20.

6 Jennett B, Bond M. Assessment of outcome after severe brain damage. Lancet. 1975; 1: 480-484.

$7 \mathrm{Lv} \mathrm{X}$, Li Y, Jiang C, et al. Potential advantages and limitations of the Leo stent in endovascular treatment of complex cerebral aneurysms. Eur J Radiol. 2011; 79: 317-322.

8 Lopes D, Sani S. Histological postmortem study of an internal carotid artery aneurysm treated with the Neuroform stent. Neurosurgery. 2005; 56: E416.

9 Katsaridis V, Papagiannaki C, Violaris C. Embolization of acutely ruptured and unruptured wide-necked cerebral aneurysms using the Neuroform2 stent without pretreatment with antiplatelets: a single center experience. Am J Neuroradiol. 2006; 27 (5): 1123-1128.

10 Mocco J, Snyder KV, Albuquerque FC, et al. Treatment of intracranial aneurysms with the Enterprise stent: a multicenter registry. J Neurosurg. 2009; 110: 35-39

11 Molyneux AJ, Kerr RS, Yu LM, et al. International subarachnoid aneurysm trial (ISAT) of neurosurgical clipping versus endovascular coiling in 2143 patients with ruptured intracranial aneurysm: a randomised comparison of effects on survival, dependency, seizures, rebleeding, subgroups, and aneurysm occlusion. Lancet. 2005; 366: 809-817.
12 Johnston SC, Higashida RT, Barrow DL, et al. Recommendations for the endovascular treatment of intracranial aneurysms: a statement for healthcare professionals from the Committee on Cerebrovascular Imaging of the American Heart Association Council on Cardiovascular Radiology. Stroke. 2002; 33: 2536-2544.

13 Bederson JB, Connolly ES Jr, Batjer HH, et al. Guidelines for the management of aneurysmal subarachnoid hemorrhage: a statement for healthcare professionals from a special writing group of the Stroke Council, American Heart Association. Stroke. 2009; 40: 9941025 .

Dr Youxiang Li

Beijing Neurosurgical Institute

Beijing Tiantan Hospital, Capital Medical University

6, Tiantan Xili, Chongwen, Beijing

100050 Beijing, China

E-mail: 13363880072@163.com 